\title{
Design and Realization of the Costas Loop
}

\author{
Xuelu Yao
}

China Satellite Maritime Tracking and Control Department, Jiangyin, 214431, China

Keywords: Costas; dynamic performance;

\begin{abstract}
When the target is in a high dynamic motion, the target sends the downlink signal Doppler and its rate of change is very large, which brings a lot of problems. The phase-locked loop is a closedloop automatic control system that tracks the phase of the input signal. In this paper, the basic principle and performance of Costas ring are analyzed, and the results of performance analysis are given. Finally, the design of software loop parameters is completed.
\end{abstract}

\section{Introduction}

Costas ring, also known as in-phase quadrature ring, is a kind of PLL (Phase Locked Loop). Since the Kostas ring has symmetry, the Kostas ring is insensitive to the phase inversion of the I and Q signals in the condition that the pre-detection integration time of the I and Q paths does not cross the modulation data.

\section{Costas Ring Phase Detector Characteristics}

Costas ring principle shown in Figure 1.

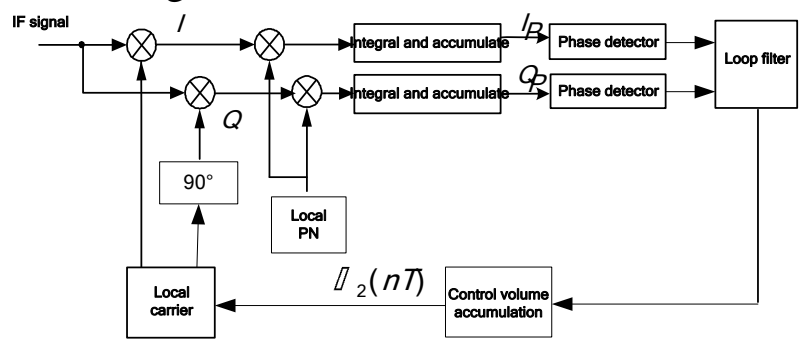

Figure 1. Costas ring schematic

Table 1 summarizes the phase errors and characteristics of the four Costas phase detectors.

Table 1. Universal Costas ring phase detector

\begin{tabular}{|c|c|c|}
\hline $\begin{array}{c}\text { Phase detector } \\
\text { algorithm }\end{array}$ & $\begin{array}{c}\text { Output } \\
\text { phase } \\
\text { error }\end{array}$ & Characteristics \\
\hline $\bar{Q}_{k} \operatorname{sign}\left(\bar{I}_{k}\right)$ & $\sin \left(\bar{\varphi}_{k}\right)$ & $\begin{array}{c}\text { High signal to noise ratio close to the best, the slope is } \\
\text { proportional to the signal amplitude, the amount of computation is } \\
\text { low }\end{array}$ \\
\hline $\bar{Q}_{k} \bar{I}_{k}$ & $\sin \left(2 \bar{\varphi}_{k}\right)$ & $\begin{array}{c}\text { Low signal to noise ratio close to the best, slope and signal } \\
\text { amplitude is proportional to the square, the amount of medium }\end{array}$ \\
\hline $\bar{Q}_{k}$ & $\tan \left(\bar{\varphi}_{k}\right)$ & $\begin{array}{c}\text { When the SNR is high and low performance is good, the signal } \\
\text { amplitude is independent of the slope. Have a higher amount of } \\
\text { computation and need to check whether or not it is 0 }\end{array}$ \\
\hline$a \tan \left(\frac{\bar{Q}_{k}}{\bar{I}_{k}}\right)$ & $\bar{\varphi}_{k}$ & $\begin{array}{c}\text { When the SNR is higher and lower when the best performance, } \\
\text { signal amplitude and slope has nothing to do with the maximum } \\
\text { amount of computing }\end{array}$ \\
\hline
\end{tabular}




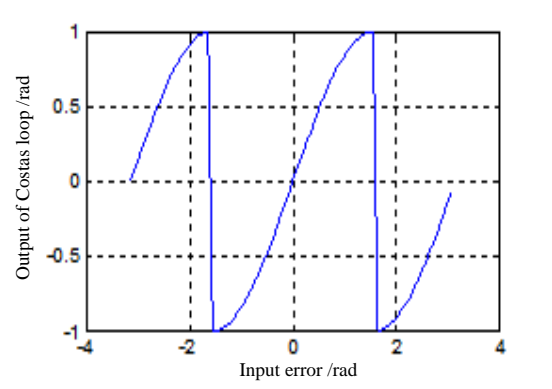

(a) $Q_{k} \operatorname{sign}\left(I_{k}\right)$

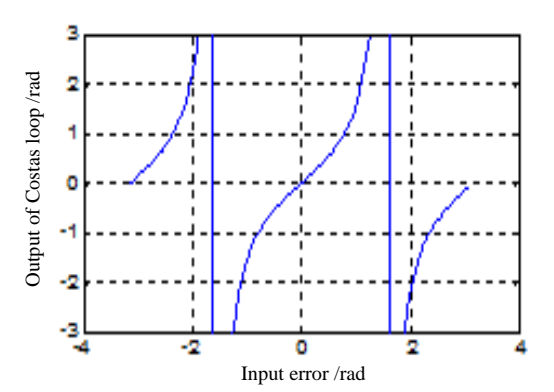

(c) $Q_{k} / I_{k}$

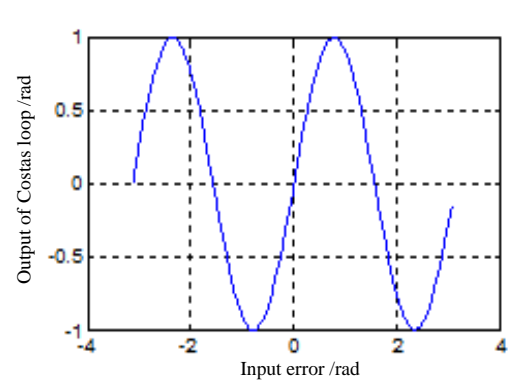

(b) $Q_{k} I_{k}$

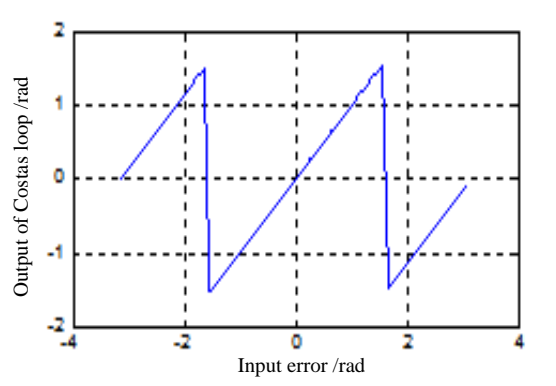

(d) $a \tan \left(Q_{k} / I_{k}\right)$

Figure 2. Comparison of Different Kinds of Phase - taking Algorithms for Costas Ring

Figure 2 shows the absence of noise interference in the case of a variety of phase recognition algorithm phase error output. If there is noise, the amplitude of the phase detector output will drop (its slope tends to be gentle), and the error curve becomes smooth at 90 degree phase error.

To DP phase detector, for example, the Kostas ring phase detector output:

$$
B_{k}=Q_{k} I_{k}=0.25 A^{2} D^{2}(k) R^{2}[\varepsilon(k)] S a^{2}\left\{\left[\Delta f_{d}(k)\right] \cdot \pi T\right\} \cdot \sin 2 \varphi_{k}
$$

$\mathrm{D}(\mathrm{k})$ is a digital symbol signal, its value is -1 or +1 , its square is 1 , so for the carrier modulation, the data symbol on the Costas loop phase identification performance without interference, that is, Data symbols are not sensitive to jumps.

According to the formula (1), when the phase of the signal is close to 0 , the phase of the loop is proportional to the error signal. The loop can be used directly to track the phase of the carrier signal. When the tracking is stable, the tracking accuracy is high However, when the phase change of the carrier signal is relatively large, it will exceed the tracking range of Costas loop, or will cause the loop to lose the lock. When the target is in high dynamic motion, Doppler will lead to the uncertainty of the phase of the carrier signal, so using Costas loop to track the phase of the carrier signal has some difficulties. So, when the target is in a static or low dynamic time, with the Costas loop for the carrier tracking, this has a high tracking accuracy.

\section{Dynamic Performance Analysis}

The dynamic introduction error is related to the loop bandwidth and the loop order. The dynamic introduction of the tracking error can be expressed as:

$$
\theta_{e m}=\frac{d^{m} \theta / d t^{m}}{\omega_{N}^{m}} \quad(\mathrm{deg})
$$

In the formula, $\theta$ is the phase expression of the carrier signal, the $m$ is the order of the phase change rate, $\mathrm{m}$ is the order of the loop, the denominator is the angular frequency of the loop, the phase error of the carrier tracking loop is the formula (2) result.

Since the carrier wavelength is $\lambda=\frac{c}{f_{R F}}=\frac{3 * 10^{8}}{2.2 * 10^{9}}=0.136(\mathrm{~m})$, the conversion of the phase unit deg corresponds to the distance unit: $360 \mathrm{deg} / 0.136 \mathrm{~m}=2647.1 \mathrm{deg} / \mathrm{m}$. Then the formula (2) can be expressed as: 


$$
\theta_{e m}=2647.1 \times \frac{d^{m} r / d t^{m}}{\omega_{N}^{m}} \quad(\operatorname{deg})
$$

The following three-stage PLL, for example, calculate the dynamic tracking threshold phaselocked loop.

Set the receiver to adapt to the maximum acceleration of $Z_{p l l} \mathrm{~g} / \mathrm{s}$, so:

$$
r^{\prime \prime \prime}=9.8 \times Z_{p l l} \quad\left(m / s^{2}\right)
$$

For the software system to achieve the tracking program, you can not consider the thermal noise error, $\theta_{e m} \leq 45^{0} \omega_{N}=1.2 B_{n}$ into the formula (4), so:

$$
Z_{p l l} \leq \frac{45 \times\left(1.2 B_{n}\right)^{3}}{2647.1 \times 9.8}=\frac{B_{n}^{3}}{360.3} \quad(\mathrm{~g} / \mathrm{s})
$$

Figure 3 shows the effect of bandwidth setting on dynamic performance under different carrier-tonoise ratios.

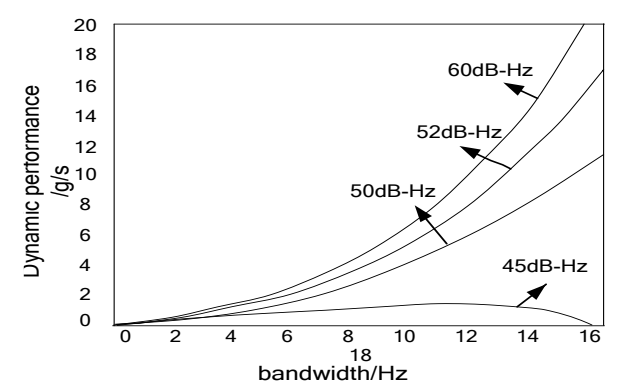

Figure 3. Effect of Bandwidth on Dynamic Performance under Different Carrier-to-Noise Ratio (T $=0.2 \mathrm{~ms}$ )

It can be seen from Figure 3: the higher the carrier-to-noise ratio, the stronger the dynamic tolerance of the loop. When the carrier-to-noise ratio is less than $50 \mathrm{dBHz}$, the dynamic performance increases with the increase of bandwidth, and the dynamic performance is the best when the bandwidth is $15 \mathrm{~Hz}$. This indicates that the tracking error introduced by the noise at low signal-tonoise ratio is very small and has little effect on the dynamic performance of the loop. When the signalto-noise ratio is low, the bandwidth increases and the SNR threshold increases, Will lose lock.

\section{PLL Design}

The second-order filter is a common loop filter inside the receiver, but this filter is more sensitive to acceleration. In a dynamic environment, the general use of third or fourth order filters. The loop transfer functions of the second and third order loop a filter is shown as follows:

$$
\begin{gathered}
T_{2}(s)=\frac{a \omega_{N} s+\omega_{N}^{2}}{s^{2}+a \omega_{N} s+\omega_{N}^{2}} \\
T_{3}(s)=\frac{b \omega_{N} s^{2}+a \omega_{N}^{2} s+\omega_{N}^{3}}{s^{3}+b \omega_{N} s^{2}+a \omega_{N}^{2} s+\omega_{N}^{3}}
\end{gathered}
$$

The corresponding noise bandwidth is:

$$
\begin{gathered}
B_{n}=\int_{0}^{\infty}\left|T_{m}(j \omega)\right|^{2} d \omega \\
T_{m}(j \omega)=\frac{c_{m-1}(j \omega)^{m-1}+\cdots+c_{0}}{d_{m}(j \omega)^{m}+\cdots+d_{0}}
\end{gathered}
$$

If you design a phase-locked loop based on software, you must digitize the loop filter. The following focuses on the calculation of the transfer function of the third-order loop filter. But with the following prerequisites:

A). Discrete domains can be achieved using bilinear transformations of continuous domains:

$$
s=\frac{2}{T} \frac{1-z^{-1}}{1+z^{-1}}
$$

B). The transfer function of $\mathrm{NCO}$ is:

$$
N(z)=\frac{k_{1} z^{-1}}{1-z^{-1}}
$$

C). The numerical form of the third-order loop filter can be expressed as: 


$$
F(z)=\frac{\left(C_{1}+C_{2}+C_{3}\right)-\left(2 C_{1}+C_{2}\right) z^{-1}+C_{1} z^{-2}}{1-2 z^{-1}+z^{-2}}
$$

D). The transfer function of the phase-locked loop is:

$$
T(z)=\frac{k_{0} F(z) N(z)}{1+k_{0} F(z) N(z)}
$$

First, the formula (11) and the formula (12) into the formula (13) available:

$$
T(z)=\frac{k_{0} k_{1} z^{-1}\left[\left(C_{1}+C_{2}+C_{3}\right)-\left(2 C_{1}+C_{2}\right) z^{-1}+C_{1} z^{-2}\right]}{1+\left[k_{0} k_{1}\left(C_{1}+C_{2}+C_{3}\right)-3\right] z^{-1}+\left[3-k_{0} k_{1}\left(2 C_{1}+C_{2}\right)\right] z^{-2}+\left(k_{0} k_{1} C_{1}-1\right) z^{-3}}
$$

Then equation (14) into equation (13):

$$
\begin{gathered}
{\left[4 b \omega_{n} T+2 a \omega_{n}^{2} T^{2}+\omega_{n}^{3} T^{3}\right]+\left[-4 b \omega_{n} T+2 a \omega_{n}^{2} T^{2}+3 \omega_{n}^{3} T^{3}\right] z^{-1}} \\
T(z)=\frac{+\left[-4 b \omega_{n} T-2 a \omega_{n}^{2} T^{2}+3 \omega_{n}^{3} T^{3}\right] z^{-2}+\left[4 b \omega_{n} T-2 a \omega_{n}^{2} T^{2}+\omega_{n}^{3} T^{3}\right] z^{-3}}{\left[4 b \omega_{n} T_{s}+2 a \omega_{n}^{2} T_{s}^{2}+\omega_{n}^{3} T_{s}^{3}+8\right]+\left[-4 b \omega_{n} T+2 a \omega_{n}^{2} T^{2}+3 \omega_{n}^{3} T^{3}-24\right] z^{-1}} \\
+\left[-4 b \omega_{n} T-2 a \omega_{n}^{2} T^{2}+3 \omega_{n}^{3} T^{3}+24\right] z^{-2}+\left[4 b \omega_{n} T-2 a \omega_{n}^{2} T^{2}+\omega_{n}^{3} T^{3}-8\right] z^{-3}
\end{gathered}
$$

The denominator of formula (14) and formula (15) are given equal:

$$
\begin{aligned}
C_{1} & =\frac{1}{k_{0} k_{1}} \frac{8 b \omega_{n} T_{s}+2 \omega_{n}^{3} T_{s}^{3}}{4 b \omega_{n} T_{s}+2 a \omega_{n}^{2} T_{s}^{2}+\omega_{n}^{3} T_{s}^{3}+8} \\
C_{2} & =\frac{1}{k_{0} k_{1}} \frac{8 a \omega_{n} T_{s}^{2}-4 \omega_{n}^{3} T_{s}^{3}}{4 b \omega_{n} T_{s}+2 a \omega_{n}^{2} T_{s}^{2}+\omega_{n}^{3} T_{s}^{3}+8} \\
C_{3} & =\frac{1}{k_{0} k_{1}} \frac{8 \omega_{n}^{3} T_{s}^{3}}{4 b \omega_{n} T_{s}+2 a \omega_{n}^{2} T_{s}^{2}+\omega_{n}^{3} T_{s}^{3}+8}
\end{aligned}
$$

Since $4 b \omega_{n} T_{s}+2 a \omega_{n}^{2} T_{s}^{2}+\omega_{n}^{3} T_{s}^{3}<<8$, the above formula can be approximated as:

$$
C_{1}=\frac{b \omega_{n} T_{s}}{k_{0} k_{1}}, \quad C_{2}=\frac{a \omega_{n}^{2} T_{s}^{2}}{k_{0} k_{1}}, \quad C_{3}=\frac{\omega_{n}^{3} T_{s}^{3}}{k_{0} k_{1}}
$$

The third - order loop form is composed of the formula (12) and the formula (19), which is used to realize the third-order phase - locked loop of the software receiver in the dynamic environment.

\section{Summary}

In this paper, the basic principle and tracking performance of Costas ring are analyzed, and the algorithm parameters of Costas ring are deduced, which paves the way for the design of Costas ring.

\section{References}

[1]. Sascha M. Spangenberg, et al. An FFT-Based Approach for Fast Acquisition in Spread Spectrum Communication System. Wireless Personal Communications, 13: 27-56, 2010.

[2]. S.Hinedi, J.I.Staman. Digital Accumulators in Phase and Frequency Tracking Loops, IEEE Trans on AES, Vo1.26, No. 1, January 2013. 